\title{
Choicing Method of Technical Combinations
}

\author{
Lijie Sha \\ Tianjin University, Tianjin 250014, China \\ Shandong University of Finance, Jinan 250014, China
}

Guojie Zhao

Tianjin University, Tianjin 250014, China

\begin{abstract}
The enterprises often encounter problems of how to make choice among several technical combinations. This article introduces a practical method --- sorting. The first is sorting method of incremental efficiency, which is based on the comparison of mean square deviations. The second is more complicated method, which is based on the comparison among efficiency index. Both are very useful in project management and production scheduling.
\end{abstract}

Keywords: Mean square deviation, Incremental efficiency, Incremental cost

\section{Problem raising}

In everyday management, the enterprises often encounter majorization solutions to technical proposals. For instance, a factory is producing two mutually supporting products $\mathrm{A}$ and $\mathrm{B}$. The inside diameter of $\mathrm{A}$ is $\mathrm{X}$ and that of $\mathrm{B}$ is $\mathrm{Y}$. The clearance between $\mathrm{A}$ and $\mathrm{B}$ is $\mathrm{Z}$, and thus $\mathrm{Z}=\mathrm{X}-\mathrm{Y}$. According to theory of probability, the mean square deviation (MSD) of the clearance $\sigma^{2} z=\sigma^{2} x+\sigma^{2} y$. The machining ways are lathing, milling and casting etc. with the clearance at $\pm 4.5 \mu$. The acceptable processing cost is 300 Yuan each set.

Table 1 indicates the processing ways, precision and cost of part A and B.

Table 1. Processing Measures, MSD and Cost of A and B

\begin{tabular}{|c|c|c|c|c|c|}
\hline \multicolumn{3}{|c|}{ A } & \multicolumn{3}{c|}{ B } \\
\hline Measures & Cost(Yuan) & $\operatorname{MSD}\left(\mu^{2}\right)$ & Measures & Cost & $\operatorname{MSD}\left(\mu^{2}\right)$ \\
\hline 1 & 165 & 36 & 1 & 100 & 16 \\
\hline 2 & 170 & 16 & 2 & 103 & 9 \\
\hline 3 & 175 & 9 & 3 & 115 & 4 \\
\hline 4 & 185 & 4 & 4 & 145 & 1 \\
\hline 5 & 200 & 1 & & & \\
\hline
\end{tabular}

The problem is the optimum choice the enterprise should make under the constraint conditions above.

This is obviously a problem of technical and economic evaluation on 20 combinations. Of course, enumeration method (it is also called mutex method) can be used to work out the mean square deviation and) the one that can satisfy the constraint conditions.

Is there any simple way to work out the satisfactory solution?

\section{Sorting method}

We believe that efficiency index or sort of incremental efficiency index can help to solve this problem.

The so-called efficiency index refers to the output of each unit of constraint resources, which is the ratio of each unit of incremental constraint resources and the corresponding increment of output. In this example, the incremental efficiency refers to the ratio of each unit of incremental cost and the decrement of MSD, which can be shown as: incremental efficiency $=$ decrement of MSD/decrement of cost. Take the processing of A as an example, if measure 2 is used instead of Measure 1, the increment of cost is $170-165=5$ (Yuan), and the decrement of MSD is $36-16=$ $20 \mu^{2}$. Therefore, the incremental efficiency $=(36-16) /(170-165)=4.0\left(\mu^{2} /\right.$ Yuan $)$. Namely, 1 Yuan of incremental cost will result in $4 \mu^{2}$ of decrement on MSD. In the same way, the numerical value of each incremental efficiency can be obtained.

Suppose $[i, j]$ represents the combination, in which $i$ stands for the processing measure sequence number of A and $j$ stands for that of B. In view of economy, the lower the cost is, the better it will be, and thus the one with the lowest cost should be chosen first. In this way, the combination $[1,1]$ is selected with total cost 265 Yuan / per set meeting 
the condition. However, there is a bigger error because $\sigma 2 \mathrm{z}=52 \mu 2$, which means $\sigma= \pm 7.2 \mu$, exceeding the consumer's requirement $\pm 4.5 \mu$. Therefore, the combination of processing measures has to be changed with more input to improve the quality. Obviously, the increased unit input should lead to the greatest decrease of MSD.

Table 2. The Incremental Efficiency of Machining A and B

\begin{tabular}{|c|c|c|c|}
\hline \multicolumn{2}{|c|}{ A } & \multicolumn{2}{c|}{ B } \\
\hline Machining Measure & Incremental Efficiency & Machining Measure & Incremental Efficiency \\
\hline 1 & --- & 1 & -- \\
\hline 2 & 4.0 & 2 & 2.3 \\
\hline 3 & 1.4 & 3 & 0.4 \\
\hline 4 & 0.5 & 4 & 0.4 \\
\hline 5 & 0.2 & & \\
\hline
\end{tabular}

From Table 2, it is obviously seen that when the machining method of Part A is changed from 1 to 2, the incremental efficiency becomes the biggest with 4.0. The second biggest is 2.3 with Part B when the machining method is changed from 1 to $2 \ldots$ The order of excellence for the combinations should be [2, 1], [2,2], ... Table 3 shows the order of excellence for all possible combinations.

Table 3. The order of excellence for the possible combinations

\begin{tabular}{|c|c|c|c|}
\hline Combination $[I, j]$ & Cost (Yuan / Each set) & MSD $\left(\mu^{2}\right)$ & Standard Deviation $(\mu)$ \\
\hline$[1,1]$ & 265 & 52 & \pm 7.2 \\
\hline$[12,1]$ & 270 & 32 & \pm 5.7 \\
\hline$[2,2]$ & 273 & 25 & \pm 5.0 \\
\hline$[3,2]$ & 278 & 18 & \pm 4.2 \\
\hline$[4,2]$ & 288 & 13 & \pm 3.6 \\
\hline$[4,3]$ & 300 & 8 & \pm 2.8 \\
\hline$[5,3]$ & 315 & 5 & \pm 2.2 \\
\hline$[5,4]$ & 345 & 2 & \pm 1.4 \\
\hline
\end{tabular}

According to Table 3, the recession curve can be drawn in Figure 1. The lines of cost restraint and MSD restraint can also be drawn there. Thus, combinations which can satisfy the restraint conditions are $[3,2],[4,2]$ and $[4,3]$. If (the) lowest cost is the target, combination [3, 2] should be selected.

By the way, the curve of incremental efficiency $[1,1] \rightarrow[2,1] \rightarrow \ldots \rightarrow[5,4]$ gives another restraint line, on which the qualified combinations are located. In other words, the rest 12 combinations are located on the upper right quadrant of the curve of incremental efficiency, composing the unqualified combinations. Therefore, the method of incremental efficiency index excludes the unqualified combinations automatically, which simplifies the decision-making process (see Figure 2).

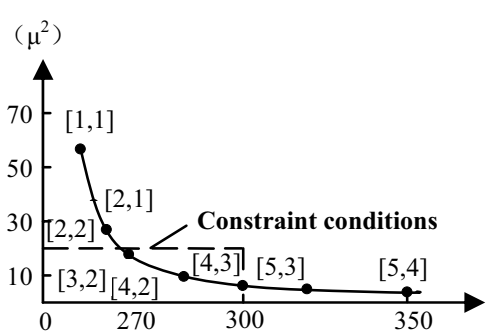

Figure 1. Solution of Marginal efficiency index sorting

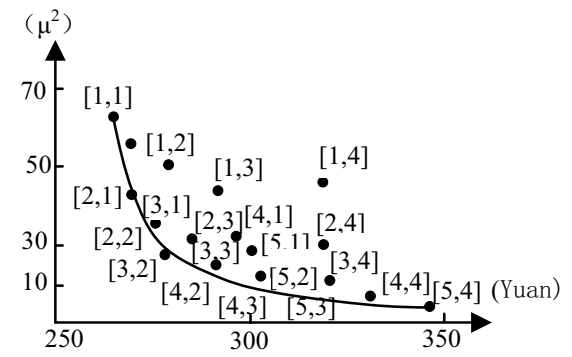

Figure 2. All the solutions and unqualified

\section{Solution to problems of complicated machining control}

Control of project and construction dispatching are problems enterprises often have to face. For example, one enterprise has to complete 3 independent machining projects I, II, III, which have to get through 3, 4, 3 machining procedures correspondingly. Five project groups $\left(\mathrm{M}_{1}, \mathrm{M}_{2}, \mathrm{M}_{3}, \mathrm{M}_{4}, \mathrm{M}_{5}\right)$ are assigned to finish the 10 procedures, 
and each group can conduct any of the 10 procedures. Table 4 gives out the time and cost each procedure requires. The cost includes fixed cost and variable cost. In the table, figures above "/" represents cost and "" stands for time needed. Each procedure has to be conducted by one group individually. The problem is how to assign the procedures to each group with better economic benefits.

Table 4. Time and Cost Needed by Each Group for Each Procedure

\begin{tabular}{|c|c|c|c|c|c|c|}
\hline Project & Procedure & $\mathrm{M}_{1}$ & $\mathrm{M}_{2}$ & $\mathrm{M}_{3}$ & $\mathrm{M}_{4}$ & $\mathrm{M}_{5}$ \\
\hline \multirow{3}{*}{ I } & 1 & (2) $30 / 16$ & (1) $25 / 15$ & $80 / 24$ & (3) $60 / 18$ & $70 / 20$ \\
\hline & 2 & (1) $60 / 13$ & $90 / 18$ & (2) $70 / 15$ & $100 / 20$ & (3) $75 / 16$ \\
\hline & 3 & (2) $60 / 15$ & (1) $40 / 13$ & $100 / 27$ & (3) $70 / 18$ & $120 / 30$ \\
\hline \multirow{4}{*}{ II } & 1 & (2) $120 / 20$ & (1) $100 / 15$ & $180 / 30$ & $150 / 23$ & (3) $135 / 18$ \\
\hline & 2 & (1) $80 / 14$ & (2) $90 / 15$ & (3) $100 / 16$ & $100 / 18$ & $140 / 20$ \\
\hline & 3 & (1) $70 / 12$ & (3) $120 / 17$ & (2) $100 / 15$ & $160 / 19$ & $130 / 19$ \\
\hline & 4 & $100 / 14$ & (1) $60 / 10$ & (3) $80 / 12$ & (2) $75 / 12$ & $85 / 13$ \\
\hline \multirow{3}{*}{ III } & 1 & (3) $50 / 18$ & (1) $40 / 14$ & $60 / 20$ & (2) $45 / 15$ & $70 / 22$ \\
\hline & 2 & (1) $45 / 13$ & (3) $70 / 24$ & $110 / 24$ & (2) $60 / 18$ & $80 / 26$ \\
\hline & 3 & (1) $35 / 18$ & (2) $45 / 20$ & $100 / 40$ & $80 / 30$ & (3) $70 / 25$ \\
\hline
\end{tabular}

It is inconvenient and unpractical to solve this kind of problems with linear programming technique. However, the method of efficiency index sort can give us very satisfying result.

First, without regard to time, mark the procedure with the lowest cost with (1), the one with the second lowest cost with (2) and the one with the third lowest cost with (3). For example, in the top line of Table 4, the order of the procedures is $\mathrm{M}_{2}, \mathrm{M}_{1}$, and $\mathrm{M}_{4}$.

Moreover, add up all the time of procedures with (1) for each group, it turns out that the time for $M_{1}$ is 70 hours, that of $\mathrm{M}_{2}$ is 67 hours and there is no time for all the others (see Table 5).

If working time is unlimited for each group, of course, it is most economical to assign all the tasks to $M_{1}$ and $M_{2}$. However, working time is usually limited. Let's suppose the longest working time for each group is 42 hours, and then some procedures have to be done by other groups.

Table 5. Combinations of the Lowest Cost

\begin{tabular}{|c|c|c|c|c|c|c|}
\hline Project & Procedure & $\mathrm{M}_{1}$ & $\mathrm{M}_{2}$ & $\mathrm{M}_{3}$ & $\mathrm{M}_{4}$ & $\mathrm{M}_{5}$ \\
\hline \multirow{3}{*}{ I } & 1 & & $25 / 15$ & & & \\
\hline & 2 & $60 / 13$ & & & & \\
\hline & 3 & & $40 / 13$ & & & \\
\hline \multirow{4}{*}{ II } & 1 & & $100 / 15$ & & & \\
\hline & 2 & $80 / 14$ & & & & \\
\hline & 3 & $70 / 12$ & & & & \\
\hline & 4 & & $60 / 10$ & & & \\
\hline \multirow{3}{*}{ III } & 1 & & $40 / 14$ & & & \\
\hline & 2 & $45 / 13$ & & & & \\
\hline & 3 & $35 / 18$ & & & & \\
\hline \multicolumn{2}{|c|}{ Time available (hour) } & 42 & 42 & 42 & 42 & 42 \\
\hline \multicolumn{2}{|c|}{ Total time needed (hour) } & 70 & 67 & & & \\
\hline
\end{tabular}

If the possible combinations and cost are calculated, it is easy to find the right choice. However, there are too many such combinations and they can not be listed for all. Therefore, we have to find a simple method to solve this problem. In order to have the lowest incremental cost, we should make the best use of $\mathrm{M}_{1}$, and thus the procedures done by other groups will take 28 hours $(70-42=28)$. If I - 2 (the second line in Table 5) is taken away, 13 hours work will be cut down from $\mathrm{M}_{1}$. If this procedure is distributed to $\mathrm{M}_{2}$, the incremental cost will be 30 Yuan (90 - 60 
=30). Accordingly, if it is given to $\mathrm{M}_{3}$, the incremental cost is 10 ; and if given to $\mathrm{M}_{4}$, the incremental cost is 40; and etc. Based on the principle of lowest cost, the best choice is to assign this procedure to $\mathrm{M}_{3}$. By the same token, it is the most economical to transfer II -2 from $M_{1}$ to $M_{2}$. If all the procedures are taken away from $M_{1}$ and given to the first alternates (with mark (2), the loss rates (rate of incremental cost and time) can be calculated as follows:

If $\mathrm{I}-2$ is given to $\mathrm{M}_{3}:(70-60) / 13=0.77 \quad$ If $\mathrm{II}-2$ is given to $\mathrm{M}_{2}:(90-80) / 14=0.71$

If II -3 is given to $M_{3}:(100-70) / 12=2.50 \quad$ If III -2 is given to $M_{4}:(60-45) / 13=1.15$

If III -3 is given to $\mathrm{M}_{2}:(45-35) / 18=0.55$

Obviously, the smaller the loss is, the better the transfer will be. Therefore, we can transfer the procedures from $\mathrm{M}_{1}$ to others with the least loss rate until there is less than 42 hours of work for $\mathrm{M}_{1}$ (III -3 and II -2 are transferred in this step). In this way, the sound result can be got (see Table 6).

Table 6. Combinations after the First Transfer

\begin{tabular}{|c|c|c|c|c|c|c|}
\hline Project & Procedure & $\mathrm{M}_{1}$ & $\mathrm{M}_{2}$ & $\mathrm{M}_{3}$ & $\mathrm{M}_{4}$ & $\mathrm{M}_{5}$ \\
\hline \multirow{3}{*}{ I } & 1 & & $25 / 15$ & & & \\
\hline & 2 & $60 / 13$ & & & & \\
\hline & 3 & & $40 / 13$ & & & \\
\hline \multirow{4}{*}{ II } & 1 & & $100 / 15$ & & & \\
\hline & 2 & & $90 / 15$ & & & \\
\hline & 3 & $70 / 12$ & & & & \\
\hline & 4 & & $60 / 10$ & & & \\
\hline \multirow{3}{*}{ III } & 1 & & $40 / 14$ & & & \\
\hline & 2 & $45 / 13$ & & & & \\
\hline & 3 & & $45 / 20$ & & & \\
\hline \multicolumn{2}{|c|}{ Time available (hour) } & 42 & 42 & 42 & 42 & 42 \\
\hline \multicolumn{2}{|c|}{ Total time needed (hour) } & 38 & 102 & & & \\
\hline
\end{tabular}

After rechecking the result after the redistribution, it is easy to find that $\mathrm{M}_{2}$ has too much to do and some procedures have to be taken away form $\mathrm{M}_{2}$ to others in the same way as above. Let's first calculate the loss rates (procedures transferred from $\mathrm{M}_{1}$ to $\mathrm{M}_{2}$ can not be returned to $\mathrm{M}_{1}$ ):

If $\mathrm{I}-1$ is given to $\mathrm{M}_{1}:(30-25) / 15=0.33 \quad$ If $\mathrm{II}-3$ is given to $\mathrm{M}_{1}:(60-40) / 13=1.54$

If II-2 is given to $M_{1}:(120-100) / 15=1.33 \quad$ If II-2 is given to $M_{3}:(100-90) / 15=0.66$

If III-4 is given to $\mathrm{M}_{4}:(75-60) / 10=1.50 \quad$ If III-1 is given to $\mathrm{M}_{4}:(45-40) / 14=0.35$

If III-3 is given to $\mathrm{M}_{5}:(70-45) / 20=1.25$

Redistribute the assignments according to the principle of least loss rate, if procedure I -1 , III -1 and II -2 are taken away from $\mathrm{M}_{2}$, and 38 hours of work is left, within the time limit. However, after the redistribution (see Table 7), tasks assigned to $\mathrm{M}_{1}$ exceed its time limit. Therefore, by the same means, some procedures have to be taken away from $\mathrm{M}_{1}$ and transferred to others except $\mathrm{M}_{2}$. The result is shown in Table 8.

Table 7. Combinations after the Second Transfer

\begin{tabular}{|c|c|c|c|c|c|c|}
\hline Project & Procedure & $\mathrm{M}_{1}$ & $\mathrm{M}_{2}$ & $\mathrm{M}_{3}$ & $\mathrm{M}_{4}$ & $\mathrm{M}_{5}$ \\
\hline \multirow{3}{*}{ I } & 1 & $30 / 16$ & & & & \\
\hline & 2 & $60 / 13$ & & & & \\
\hline & 3 & & $40 / 13$ & & & \\
\hline \multirow{4}{*}{ II } & 1 & & $100 / 15$ & & & \\
\hline & 2 & & & $100 / 16$ & & \\
\hline & 3 & $70 / 12$ & & & & \\
\hline & 4 & & $60 / 10$ & & & \\
\hline
\end{tabular}




\begin{tabular}{|c|c|c|c|c|c|c|}
\hline \multirow{2}{*}{ III } & 1 & & & & $45 / 15$ & \\
\cline { 2 - 7 } & 2 & $45 / 13$ & & & & \\
\cline { 2 - 7 } & 3 & & & & & $70 / 25$ \\
\hline \multicolumn{2}{|c|}{ Time available (hour) } & 42 & 42 & 42 & 42 & 42 \\
\hline \multicolumn{2}{|c|}{ Total time needed (hour) } & 54 & 38 & 16 & 15 & 25 \\
\hline
\end{tabular}

Table 8. Combinations after the First Transfer

\begin{tabular}{|c|c|c|c|c|c|c|}
\hline Project & Procedure & $\mathrm{M}_{1}$ & $\mathrm{M}_{2}$ & $\mathrm{M}_{3}$ & $\mathrm{M}_{4}$ & $\mathrm{M}_{5}$ \\
\hline \multirow{3}{*}{ I } & 1 & $30 / 16$ & & & & \\
\hline & 2 & & & $70 / 15$ & & \\
\hline & 3 & & $40 / 13$ & & & \\
\hline \multirow{4}{*}{ II } & 1 & & $100 / 15$ & & & \\
\hline & 2 & & & $100 / 16$ & & \\
\hline & 3 & $70 / 12$ & & & & \\
\hline & 4 & & $60 / 10$ & & & \\
\hline \multirow{3}{*}{ III } & 1 & & & & $45 / 15$ & \\
\hline & 2 & $45 / 13$ & & & & \\
\hline & 3 & & & & & $70 / 25$ \\
\hline \multicolumn{2}{|c|}{ Time available (hour) } & 42 & 42 & 42 & 42 & 42 \\
\hline \multicolumn{2}{|c|}{ Total time needed (hour) } & 41 & 38 & 31 & 15 & 25 \\
\hline
\end{tabular}

Though we can just get an approximate result, yet it is a very satisfactory method in practices. It is worth using in project management and production scheduling.

\section{References}

Jin, Ruiming. (1990). Technical Economics. Tianjin Nankai University Publishing House.

Zhao, Guojie. (1999). Technical Economics. Tianjin University Publishing House. 